\title{
PERSPECTIVES OF THE TOOTH RESTORATION TECHNOLOGY BASED ON THE COMPUTED TOMOGRAPHY DATA
}

\author{
Maxim Putrik*, Vladimir Ivanov, Igor Antsygin \\ Federal State Autonomous Educational Institution of Higher Education "Ural Federal University \\ named after the first President of Russia B.N. Yeltsin", 620000, Ekaterinburg, Russia
}

\begin{abstract}
The aim of the study is to create an image processing system, which allows dentists to create tooth surface automatically. X-rays images of jaws from the cone beam tomography or the spiral computed tomography and images from the micro-computed tomography are the initial data for processing. Example of using the combination of optical and $\mathrm{x}$-ray images in the dentistry is described. Also, it was shown how to use the microcomputed tomography method to plan the placement of orthopedic structures on the lost teeth sites.
\end{abstract}

\section{Introduction}

Computed tomography is a method of examination, which is widely used in medicine, in particular, in dentistry at present. This method allows you to provide the doctor the exhaustive information which is necessary for diseases diagnosing and treatment planning.

Modern equipment for CT scanning is computer tomographs, among which there are two types:

- spiral computer tomograph;

- cone beam computer tomograph (only for examinations of the maxillofacial area).

Appearance of the above types of apparatus is shown in Figure 1.
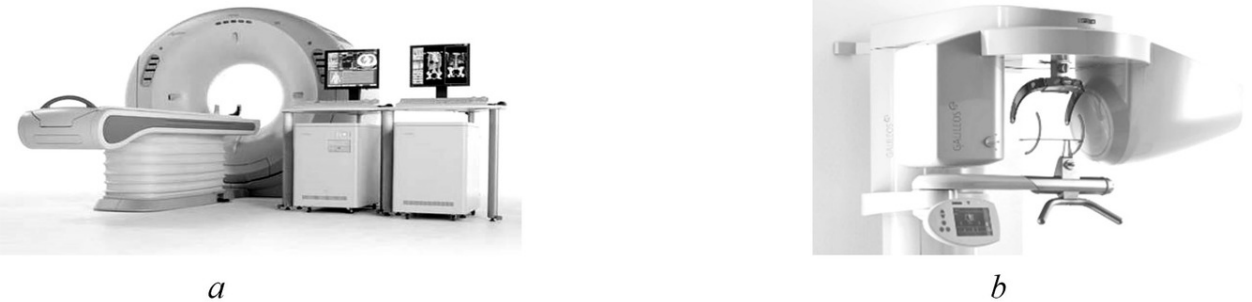

Fig. 1. Types of computer tomographs: a - spiral computer tomograph Aquilion (Toshiba, Japan); $\mathrm{b}$ - cone beam computer tomograph (Sirona GmbH, Germany).

*Corresponding author: pmb-88@mail.ru 
The physical principle of slice formation, which is used in a spiral computer tomograph, is following: the detectors of ionizing radiation are located on the ring, the emitter (X-ray tube) moves inside this ring and generates a pulsed X-ray beam. The patient is located inside this ring on a special bed, which moves inside this ring. As a result of simultaneous rotation of the ring and motion of the bed, a set of digital signals is formed on linear detectors (after passing an X-ray beam through the patient's body), which correspond to the $\mathrm{X}$-ray images of the body section in various projections. Then the inverse Radon transform is applied, the essence of which is to determine the function of two variables (the $\mathrm{X}$-ray image of the body section) over the set of linear integrals (the set of projections) from this function. For the subsequent formation of a three-dimensional image, a set of planar images is used. The determination of the density of human body tissues on the cross-sectional image obtained on a spiral computer tomograph is carried out by means of the use of the Hounsfield scale, based on the character of the interaction of X-ray radiation with matter. The attenuation of X-ray quanta in matter is described by an exponential law.

The section image is formed in gradations of gray color, in accordance with the value of HU (Hounsfield units). For example, areas on the cut with a high HU (bone image) will appear brighter on the monitor screen than soft tissue areas that have a low HU.

When forming a section image on a cone beam computer tomograph, a different physical principle is used. The emitter (X-ray tube) generates a conical shaped pulse beam that passes through the skull of the patient and is fixed to a planar detector that is much larger than the linear detectors of a spiral computer tomograph. Thus, almost all the maxillofacial area is covered in one scan. The volumetric image is formed after scanning a series of planar images at different positions of the emitter and the detector. However, in the case of cone beam tomographs, relative units are used instead of Hounsfield units, in accordance with which for air the luminance value is 0 , and the tissue with the maximum density in the special calibration phantom has a luminance value 4095, for example.

The DICOM (Digital Imaging and Communications in Medicine) standard is used to record digital medicine data. The file of this format contains not only a matrix with an image, but important information of a diagnostic nature. The file itself is divided into socalled "Information Elements" (Date Element), which are formed into separate groups. Thus, access to the "atom" of information is carried out by two numbers: the group number and the number of the element within the group. This standard is designed to make medical information independent of device manufacturers. Attached information about the survey and a snapshot make it possible to adequately and simply present the data to the doctor. In the event that the program can not recognize any attribute (element) of the file, it should skip it - for example, not all devices can read and, accordingly, write patient's coordinates to a file, there may also be problems with recognizing Cyrillic characters.

Modern X-ray computer tomographs make it possible to obtain images with a pixel size around $300 \mu \mathrm{m}$ and software designed to analyse survey results is used to build various sections, synthesize three-dimensional models, virtual operations planning, etc.

Nevertheless, the pixel size of images around $300 \mu \mathrm{m}$ is not sufficient to solve the modern dentistry problems. That's why the method of microcomputed tomography [1] gradually spreads. It is used for the analysis of techniques for tooth caries removing, assessment of bone tissue density, and studying the structure of the root canals. Images from a microcomputer tomograph have a pixel size from $1 \mu \mathrm{m}$ and are comparable with optical images in quality [2]. The disadvantages of the microcomputed tomography method are following: a long scan time (several hours), the possibility of studying small samples in vitro only.

Consider the perspectives of using this method for in vivo research. The images which are necessary for the analysis of the internal teeth structure and jaw bones will be obtained 
after the examination of the maxillofacial area. Also, 3D tooth surfaces synthesized on the basis of these images can be used to model temporary crowns and inlays.

\section{Combination optical and x-ray images}

The use of the microcomputed tomography method will allow planning the placement of orthopedic structures on the lost teeth sites. An example of such technology [3] (crown restoration of a lost tooth) is realized in the line of CEREC devices (manufactured by Sirona, $\mathrm{GmbH}$ ): the patient's oral cavity can be scanned by an intraoral camera or dental models made from special gypsum can be scanned too [3]. The essence of this technology lies in the construction of three-dimensional surfaces of jaws (upper and lower). The surface of the lower jaw is scanned to visualize the site of the lost tooth and then to model the dental crown at this site. The surface of the upper jaw is scanned in order to match the surfaces of the modelled crown and the crown of the antagonist tooth: the newly created tooth crown should be modelled not to interfere with the existing teeth. After the crown is modelled, it's image is transferred to a special machine for subsequent production from ceramic blocks.

This technology has the following drawbacks. Modern intraoral cameras allow you to scan without using a special powder, which is sprayed in the mouth and unevenly settles on the teeth. However, the dental row is unevenly covered with saliva when scanning.

Scanning of the dental model made of special gypsum can be performed both by a special scanner and an intraoral camera. It should be noted that the tooth model is modelled manually on the basis of the impression of the patient's jaw. Thus, the quality of the performed manual manipulations has a great influence on the final result.

Planning an implant operation is one of the stages in the process of restoring a lost tooth. Currently, implantation operations are performed only after the virtual planning is carried out, which involves placing the implant model inside the jawbone image: the future implant must be sufficiently immersed in the jawbone, and its axis must pass through the centre of the planned orthopedic structure. To visualize this process, Sirona company implements the possibility of importing optical images (tooth crown) into the result of scanning the maxillofacial area performed on a computer tomograph [3].

Let's describe the virtual planning of the implantation operation [3]. Since the results of the examinations of the maxillofacial area performed on a cone beam computer tomograph GALILEOS are used in this study, the example of virtual planning will be considered for the SIDEXIS software with the additional module GALILEOS Implant (supplied with the GALILEOS computer tomograph).

The SIDEXIS software (see the Figure 2), in addition to the usual viewing of survey results in all planes, allows performing additional constructions, namely: drawing a neural canal and performing a virtual implantation operation. To draw a body of a nerve channel, a dentist or a radiologist on several transverse sections marks the location of the nerve channel in the bone, after the program draws the entire channel in all the pictures. For the virtual implantation operation, first of all, it is necessary to analyze the condition of the bone tissue in place of the missing tooth and choose the number of the tooth. After that, the program will suggest choosing the appropriate implant from the database. The parameters of the implant are its diameter and height, but the implant has a rather complex shape resembling a paraboloid and a ribbed outer surface for secure anchoring in the bone. In the event that the required implant is not in the database, it is possible to create a threedimensional model, having previously indicated the diameter and height. The simulated implant will take the form of a regular cylinder. 


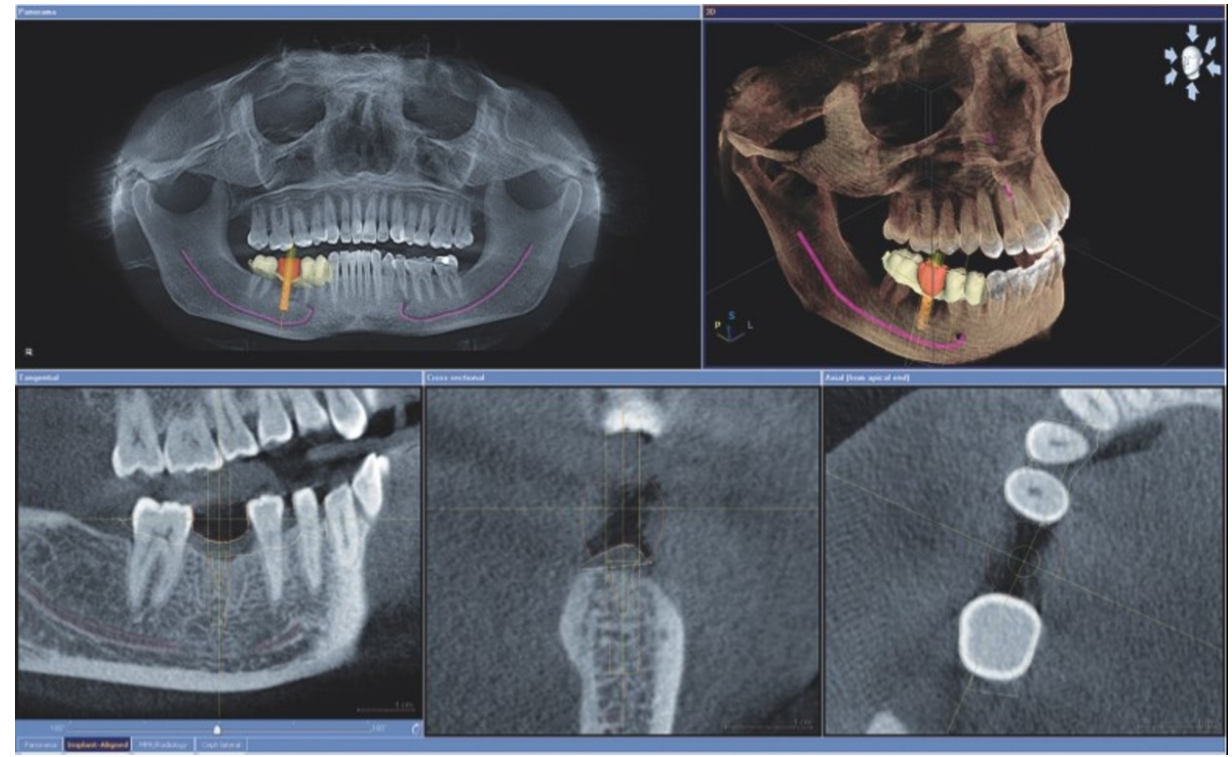

Fig. 2. Virtual implantation operation.

After selecting the implant, its image will appear on the current sections (Figure 2). To move the implant, it is enough to pull it with the mouse, holding the left key, and at the position of the mouse pointer near the implant axis, it is possible to rotate around one of the bases, thus planning the location of the real implant in the jawbone. Moving and rotating the implant are also possible in any of the sections, and changing the coordinates of the implant causes all images to be updated. When several implants are staged, the active implant is displayed in red, and the rest - in white.

\section{Use of the micro-computed tomography data}

For patients who have dentist's recommendation to remove of one of the teeth, the author suggest a different way of modelling the surface of the crown in place of tooth to be removed. It is necessary to make a survey of the patient's maxillofacial area on a computer tomograph in order to confirm the indications for tooth extraction. If such indications are confirmed, tooth extraction is performed, and the removed tooth is subjected to scanning on a microcomputer tomograph [4] whose detector resolution is significantly higher than the resolution of a CT scanner with a cone beam (CBCT). The removed tooth was scanned at the SkyScan (BRUKER-MICROCT, Belgium) with following parameters: voltage $50 \mathrm{kV}$, $23 \mu \mathrm{m}$ pixel size, $11 \mathrm{Mp}$ x-ray detector resolution.

To determine the tooth boundaries (on the images from microcomputer tomograph) using Gaussian filter the following method is used [5]. To apply this method, it is necessary to set the brightness value of pixels corresponding to the image of healthy tooth. It was found experimentally that this brightness value is no less than about $400 \mathrm{HU}$. Let's consider the mentioned method in detail. The Gaussian filter is applied on the original image at first (see Figure $3 a$ ) to increase signal-to-noise ratio (see the result at Figure $3 b$ ). Then pixel brightness values are read from the left, then from the right side and upper side of the image. When the pixel brightness value of the health tooth (400 HU or more) is determined, the border point of the tooth is assigned. Points found in this way are highlighted in white in Figure $3 c$, where the thick boundary is depicted. 


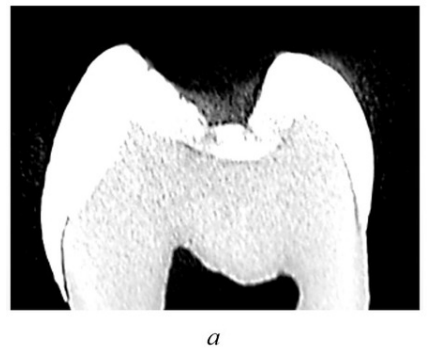

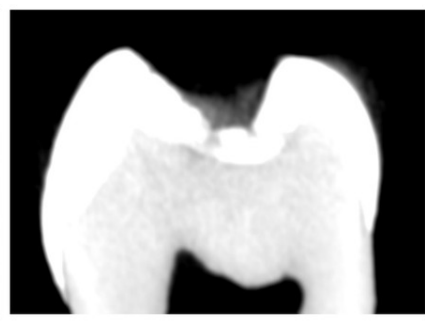

$b$

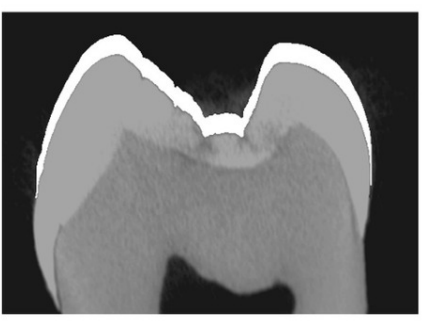

$c$

Fig. 3. High resolution tooth cuts: $a$ - original image; $b$ - original image after Gaussian filter application; $\mathrm{c}$ - tooth boundary is depicted in white colour.

The next step is to unite all boundary lines to create an entire tooth surface. The surface of the removed tooth obtained in this way is comparable in quality to the image obtained with the use of a three-dimensional intraoral optical camera. Further, the obtained surface (Figure 4) of the removed tooth is used to model and manufacture an artificial tooth that will be placed in site of the removed tooth [2].

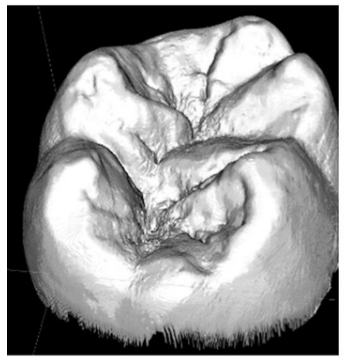

$a$

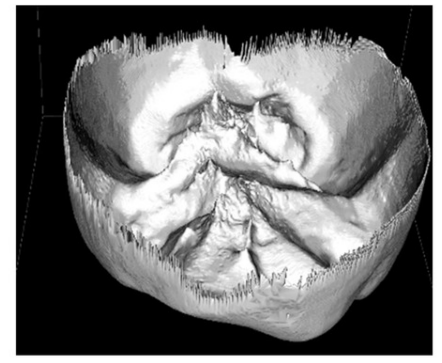

$b$

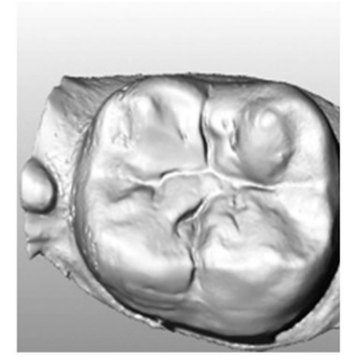

$c$

Fig. 4. High resolution tooth surface images [2]: a - tooth surface impression, obtained on the basis of microcomputed tomography data; $b$ - tooth surface impression, obtained on the basis of microcomputed tomography data (inside view); $\mathrm{c}$ - optical image.

It is assumed that it is possible to import the image obtained on a microcomputer tomograph in the result of examination of the maxillofacial region.

The presence of the patient is not required, "manual" work is excluded, and therefore manufacturing errors are eliminated, the labour costs of the doctor are significantly reduced, and the process of modelling the dental crown is performed automatically with this approach (the doctor can correct the resulting surface if necessary).

The tooth surface at Figure 4 was used for manufacturing by 3D printer from biocompatible material (A3 colour at Vita scale), see the Figure 5. 


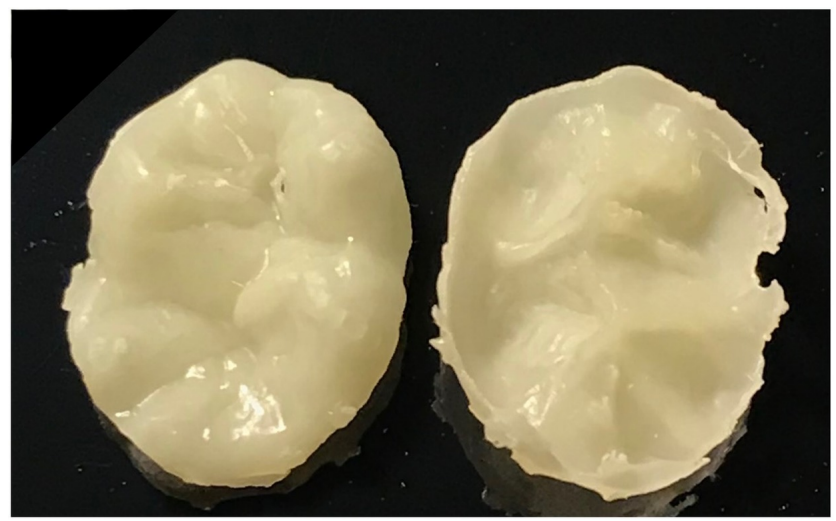

Fig. 5. Printed high resolution tooth surface.

\section{Conclusion}

Predicting further progress in the technology of the production of computer tomographs in terms of increasing the resolution of images, we can conclude that the surgical guides [3], based on high resolution images, will allow doctors to make accurate and safe implantation operations.

\section{Acknowledgements}

The research presented in this paper was supported by the Russian Foundation for Basic Research (grant No 16-29-04388/17).

\section{References}

[1] M. Naitoh, A. Katsumata, S. Mitsuya, The Int. J. of Oral \& Maxillofacial Impl. 19, 239 (2004)

[2] M.B. Putrik, J.E. Lavrentyeva, V.YU. Ivanov, News of High. Educ. Instit. Phys. (in Russian) 57, 70 (2014)

[3] A.N. Ryakhovsky, Digital dentistry (in Russian) (LLC "Avantis ", Moscow, 2010)

[4] Skyscan 1272: desk-top high-resolution micro-CT, [Online] URL: http://brukermicroct.com/products/1272.htm.

[5] M.B. Putrik, Y.E. Lavrentyeva, I.N. Antsygin, Biomed. Eng. 48, 135 (2014) doi: $10.1007 / \mathrm{s} 10527-014-9437-y$ 